\title{
The Need for General Collective Intelligence in Addressing the Sustainable Development Goals
}

\author{
Andy E. Williams (awilliams@nobeahfoundation.org)
}

\begin{abstract}
Background: This study explores the factors constraining group decision-making, where those factors might tend to restrict groups from selecting optimal solutions with regards to sustainability or sustainable development. This study also explores the factors constraining group decision-making from framing problems in the optimal way (choosing the optimal problem to solve), and how together these factors constraining groups from choosing the optimal problem to solve or the optimal solution to solve it with, might tend to bar groups from achieving optimal outcomes. To address the first set of constraints, collective intelligence algorithms aim to use the intelligence of crowds to select optimal solutions. To address the second set of constraints, General Collective Intelligence solutions, as defined in this paper, aim to further improve outcomes by selecting the optimal problem to solve.

Results: In the absence of General Collective Intelligence fundamental economic forces are suggested to drive a continual increase in the alignment of group decision-making with the interests of decisionmakers rather than with optimizing collective impact. And while sustainability and sustainable development might be achieved locally in the face of this misalignment, globally this misalignment is suggested to compete directly against that achievement. For this reason, maximizing collective outcomes such as impact on sustainability or sustainable development is suggested to require General Collective Intelligence in order to reliably increase the forces driving groups towards prioritizing collective impact until those forces are greater than the forces driving this misalignment. As a result, without General Collective Intelligence this misalignment of group decision-making in a direction other than optimal collective impact is suggested to be a hidden "bug" that may prevent sustainability and sustainable development from being reliably achievable globally through current sustainability or sustainable development programs.
\end{abstract}

Conclusions: General Collective Intelligence is a pattern of biomimicry that potentially replicates the robust and stable multi-cellular cooperation nature has evolved over more than a billion years to enable cells in organisms to cooperate to sustainably achieve outcomes. Platforms organizing groups into such a General Collective Intelligence are suggested to be required in order for sustainability or the sustainable development goals to be reliably achievable globally.

\section{Introduction}

A General Collective Intelligence (GCI) is proposed here to be a system of collective cognition with general problem solving ability, and therefore potentially vastly greater ability to address collective problems. Just as general problem solving ability (true intelligence) in individuals requires the capacity for reasoning so the individual might potentially solve any problem they can conceive, general problem solving in groups implies the group can collectively follow or execute a single line of reasoning, using any reasoning processes available to the group, giving the collective intelligence the ability to potentially solve any problem it can conceive as well. This collective intelligence differs from the intelligence of individual members of the group in that just as individual intelligence is fundamentally aligned with the individual's well-being, GCI is fundamentally aligned with collective well-being. A recently defined human-centric Functional Modeling Framework (FMF) proposes to define all the functions required for this collective cognition [7]. This framework defines a GCI to be a system of collective cognition that implements all these functional components. Recent papers propose such a model of GCI that has been defined within this framework [1], [8].

\section{Constrained to Solving the Wrong Problem Without GCI}


Many in sustainable development have pointed out the difficulties that arise from identifying the wrong problem [2],[3]. But the theory behind this model of GCI suggests that without GCI groups will in many cases not only tend to solve the wrong problem, but also tend to be constrained to do so. To understand why, imagine there is a famine that has left you and your family starving in South Sudan. Somewhere in Brussels, a program manager at a large humanitarian organization is deciding on what aid to send in response. Before deciding to send that aid, the program manager might go over the organization's long list of policies and procedures, perhaps making sure that any food aid meets the recommended minimum daily allowances of nutrients, perhaps making sure it comes from an approved supplier, perhaps making sure it comes from factories that meet its labor standards, and so forth, before finally sending three days worth of food aid after that process is concluded. Rather than considering all of this, you in South Sudan on the other hand might have preferred to just take the money equivalent to that three days worth of aid. Anything big donor organizations do is expensive. So with that money you might have been able to simply buy a month's worth of cornmeal flour to make ugali (a type of cornmeal porridge) from a passing trader that same day. Ugali is largely starch, and might not meet the donor organization's daily nutrient requirements. But it is familiar to the primarily starch based diet in rural Africa where meat is harder to come by [17], and will protect your family from starvation.

Or you might have preferred to take the money for that three days worth of aid and simply travel with your family to stay with relatives in neighboring Kenya, where instead of eating for three days, you and your family might be fed and sheltered for the next six months. In this way both of these options might result in levels of food security that are an order of magnitude or more greater. This example is somewhat contrived and may not represent the actual policies of donor organizations or the actual possibilities open to victims of famine. But when trying to maximize outcomes, this hypothetical example shows the importance of decentralizing decision-making to put some control about decisions over interventions into the hands of those impacted by those outcomes. Because each of these people is solving a different optimization problem. The person in the famine is solving the problem of the famine. The program manager is solving the policies of his organization. Centralizing decision-making in the hands of one entity is one of the many ways groups can be constrained to solving the wrong problem.

\section{Perverse Incentives for Collective Intelligence Solution Providers Without GCI}

Collective intelligence aims to find optimal solutions. But the only way for a provider of a collective intelligence decision-making solution to be commercially successful in cases such as that above might be to "sell" the decision-making solution to the party with the money, in this case aid providers such as this donor organization. This may be true whether the collective intelligence solution provider is a nonprofit and in requesting grants must sell their pitch to donor organizations, or whether the solution provider is for-profit and must sell their solution to donor organizations literally.

In order to be successful, a collective intelligence solution provider would then need to design a decision-making system that selects the solution with optimum fitness in solving the organization's policies rather than optimum fitness in solving the actual problem. In other words, in order to have the capacity to solve the problem the solution provider would need to understand why collective intelligence might be required to do so. But in order to be successful in the market, they might have to focus on the donor organization's policies instead. If prioritizing collective intelligence is indeed critical to solving the actual problem, this focus elsewhere implies either not understanding the importance of collective intelligence and unknowingly promoting a decision-making approach with a fatal flaw that could prevent it from solving the problem, or understanding the importance of collective intelligence and intentionally deciding not to solve the problem. Because in any scenario involving centralized decision-making by a donor organization such as the one responding to the crisis above, this 
model of such centralized decision-making suggests that solution providers who attempt to actually solve the problem might tend to fail to successfully compete for the resources required to sustainably deliver solutions and to scale deployment of those solutions, regardless of good intentions.

For these reasons, any centralized decision-making process in which the donor organization has sole ownership of identifying the problem may be likely to be solving the wrong problem. And when the problem and solution spaces are large, if solutions are chosen through policies the organization has defined, they may be likely to be solving the wrong problem with the wrong solution regardless of which solution they choose. For example, if the donor organization had chosen the policy of trying to source food aid from passing traders, there may be individuals for whom importing food aid is the optimal solution, so the donor might still be wrong. Just like last week's lottery winning number is likely to be the wrong one next week because there are so many potential winning combinations, optimal definitions of problems and optimal solutions differ for each person being assisted and are constantly evolving as the situation changes. When speaking of large numbers of people, trying on one's own to predict the optimal intervention for each individual might be considerably less successful than a process that in effect asks each individual what the winning number in their individual lottery actually is.

On the other hand the policies of organizations are created to ensure organizations conduct their operations in a consistent way [9], [10]. But because modeling and optimizing policies is difficult and the results sometimes unpredictable [11], [12], this consistency might be less like the consistent flow of water to fill every space where it is needed, than the consistent position of a telephone pole embedded in concrete that would be better placed somewhere else. As anyone can attest after having spent time lost in an automated phone assistance system with an issue that doesn't match the available options, such rigidity might force organizations to conduct themselves in a way that is a poor match with some customer problems. However, without policies, whether grant-making policies, human resource policies, or for the insurance industry, without the payment policies they sell as insurance, then due to factors such as risk of legal liability, organizations as they currently exist, particularly large ones, might struggle against chaos and ambiguity to gain the capacity to coordinate their operations to address any problems at all [18].

As mentioned, in the FMF the general collective intelligence of organizations and other groups is distinguished from the intelligence of individuals in that individual intelligence is assumed to be fundamentally aligned with solving problems related to the individual's well-being. General collective intelligence is defined in the FMF as being fundamentally aligned with solving problems related to collective well-being. If it is true that GCI is required to create intelligence that is aligned with collective well-being, any inability of organizations to operate without policies might not arise from the intrinsic importance of policies [13], but from the lack of GCI. Individuals, for example, might very comfortably go without policies to govern themselves in most of their own activities. Perhaps because their individual intellect is reliably aligned with their well being as an individual, rather than needing any policy to deal with the promise of every fortunate event or the risk of every adverse one, an individual might deal with such uncertainty by understanding that when the time comes, their intelligence can be relied upon to make whatever decision it concludes promises to maximize outcomes for them.

General collective intelligence in addition to policies, rather than policies alone, may be a better solution. Because achieving alignment with collective well-being in all cases might require the group's problem solving ability to be truly general, and therefore distributed throughout all processes, not just in the policy layer. And taking the definition of GCI provided by the FMF, collective general problem 
solving ability implies GCI. Without a system of GCI to coordinate decision-making across all processes, even having a system of collective intelligence driving decision-making in some processes might not be enough to avoid the spread of any tendency for alignment with individual pet interests or agendas [14] rather than with objectively measurable collective outcomes. Alignment with individual pet interests or agendas might be expected when any facet of decision-making is less driven by collective intelligence, and more driven by the individually aligned intelligence of those in leadership who set policies.

\section{Current Barriers to Solving Collective Problems Without GCI}

Without a methodical system for doing so, a number of barriers to optimizing the outcomes of collective decision-making exist:

\section{Some Barriers to Optimizing Collective Decision-Making}

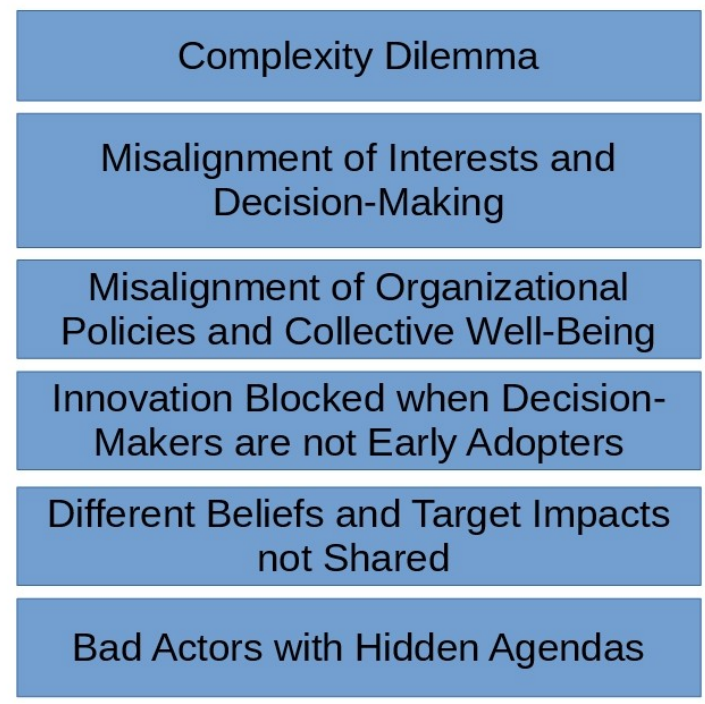

As an example, there is the complexity dilemma in which solutions with the greatest potential for impact are routinely excluded from consideration if they are considered by the group to be too complex [4].

\section{The Root of the Problem}

The FMF, and therefore the model of GCI derived from it that is discussed in this paper, have a hierarchy of adaptive domains in which they might adapt to solve problems proposed by the environment. One is the domain for adapting through reasoning, which consists of a number of cognitive functions that can be used to construct any reasoning processes. Another is the domain for adapting through cooperation, which consists of a number of cooperative functions that can be expressed more simply as principles. These principles are that any group of individuals that must cooperate in any group to achieve any collective goals, are required to cooperate according to welldefined principles of decentralization in order for that cooperation have the capacity to reliably maximize outcomes for all participants involved [5]. And the model assumes that in order to maximize the overall outcome, cooperation must be organized within well-defined principles of centralization which prioritize functionality that has greater impact. But the improved problem solving of collective intelligence isn't useful if it isn't used. Rather than imposing rules that can be broken, the principles of intelligent cooperation in this GCI model are designed to adapt to use whatever contribution from any 
participant that works best, so that it naturally converge on adopting whatever works to balance this centralizated prioritization of single functions and decentralized maximization of collective outcomes. In doing so, it maximizes the degree that the overall cooperation is sustainable.

Assuming that the possible modes of interaction between individuals or groups are cooperation, competition, and co-existence (if doing neither), game theory based modeling of this interaction between centralization and decentralization in the execution of group processes [5] suggests that without general collective intelligence to provide the pervasive decentralization required to balance any natural tendency towards centralization, impact on entire classes of collective goals might not be reliably achievable. Because without GCI, this modeling suggests that competition naturally centralizes decision-making, and unbalanced centralization of decision-making creates perverse incentives that act as a barrier to choosing optimal solutions.

One potential mechanism for unbalanced centralization is that for individual entities and groups that are allowed to either compete or cooperate, technology can provide competitive advantage. Individual entities and groups might neither compete or cooperate, but might simply coexist. For example, nations might not allow their defense contractors to compete for the business of rival countries, or to cooperate with other companies in those countries. But when competition is allowed, this competitive advantage can potentially be used to win more resources, to then buy more technology, in a cycle of increasing inequality. While disruptive new technologies might dramatically shift competitive advantage to new players, this concentration of resources helps ensure those with market dominance can acquire both new technologies and/or the services of experts in any new technology. In addition, once competition has created a market for a product or service (for example a market for cars as opposed to a market for more walkable cities), the new winner does not start from zero. The market grown by the previous winner can potentially provide a considerable resource for the new winner to take over. So markets may still tend towards increasing this cycle of inequality for some entity, even if not the same one. Although inequality trends differ considerably depending on the concept used, some studies do show the constant increase in the global GINI coefficient (a measure of inequality) over all human history for which data is available, which appears to support the existence of such systemic forces [15].

Competition and inequality are not intrinsically bad. In the natural world they are essential to the natural selection that makes ecosystems more fit. The issue is that technology removes the limits to our consumption from being what our appetites can hold, or what we can physically store or carry. Removing the limits to inequality potentially means that organizations might never be allowed to stop competing to unsustainably increase the consumption of their customers so they can acquire further resources, without the threat of being eliminated from the marketplace by others who will do so in their place. Where such a technology based capacity for unbalanced centralization might force for-profit organizations to compete to increase the consumption of their customers, in non-profit organizations this capacity for unbalanced centralization might force similar competition. In the case of non-profits, this could potentially force the organizations to compete for donations, rather than cooperating to maximize any collective maximize impact such as sustainability or sustainable development.

In summary, without adherence to these principles of decentralized cooperation within a framework of centralized cooperation, game theory modeling [5] suggests that not only might decision-making in human groups naturally tend to be sub-optimal for maximizing collective well-being, but that a tendency towards alignment with the interests of a continually decreasing minority might even prevent the solution to the problem. 
This problem might be pervasive. Every product or service involves an entire life-cycle of processes from research and design, to manufacturing, servicing and recycling. If group processes do have a tendency towards centralization without GCI to provide the capacity for a balanced amount of decentralized decision-making in all processes, then some processes related to a product or service may be centralized in a way that is sub-optimal for collective well-being, even for a product or service that is otherwise decentralized. This means also being sub-optimal for impacts related to collective wellbeing, such as sustainability, or scaling sustainable development. To achieve this balance, GCI provides several layers of decentralization [8], without which processes might be centralized within each of these layers. This suggests that without GCI, all decision-making related to any product or service might tend to have some degree of unbalanced centralization, because the mechanisms required for decentralization of at least some cooperation processes are simply lacking.

Because the several layers of decentralization provided by GCI are proposed to be required to achieve this balance, this constraint driving groups to solve the wrong problem is proposed to be a bug in all collective decision-making processes without GCI. To the degree that these layers of decentralization are not known by decision-makers in any process, the corresponding mechanisms of centralization the layers are meant to balance out might not be apparent at all, making the resulting centralization not only a bug, but an invisible bug in sustainable development and sustainability initiatives.

Through this trend towards centralization, if this centralization is unbounded, then an ever increasing ability of the process owner (in this case the provider of any product or service) to shift resources and decision-making so that it drives decisions towards serving their interests might act as a "tax" that steadily shifts resources towards increasing this imbalance. Through a trend towards centralization in the private sector, resources might be shifted between rivals as the result of direct competition. In the non-profit sector resources might be shifted as the result of organizations competing for funding rather than cooperating to maximize impact on collective challenges. In the case of parties that interact with a third party through a product or service of that third party, if alignment with that third party's interests forms a "tax" that continually extracts invisible value from any interaction between the first two, whether competition or cooperation, the shift in resources to that third party might be less visible. Since this extracted value is not aligned with the interests of either of the two parties as consumers, it is not necessarily paid in exchange for making the product or service better, and therefore might act as a kind of rent-seeking. In any case, such a pervasive shift in resources could form an invisible force driving inequality in the favor of an ever-decreasing few.

In this sense, the problem being solved by centralized decision-making is not maximizing the fitness of any product or service in achieving collective well-being for themselves and the customer, but maximizing the ability of the process owner to acquire resources and compete, potentially through driving an unsustainable increase in consumption that might generally harm collective well-being. So simply because technology provides the capacity for greater and greater inequality, without general collective intelligence to ensure collectively intelligent cooperation by decentralizing processes where needed, centralization might result in all processes tending towards ever increasing inequality that decreases fitness in achieving collective well-being, so that for at least some problems (those for which optimal solutions don't align with the interests of decision-makers) collective well-being may not be reliably achievable. And because the problem space and solution space becomes more larger and optimal solutions more sparsely distributed for larger groups, misalignment with what decision-makers perceive to be their interests may become more likely as groups get larger.

Defining simple functional models of processes as having inputs, outputs, and other information we define as the context of the process' execution, then any tendency of processes to centralize ownership 
of themselves or other processes through driving increased consumption of resources suggests inputs and outputs of some processes must become progressively more unbalanced. If so, because centralization constrains decision-making as described above, this suggests that collective impacts like the Sustainable Development Goals may not be reliably achievable without GCI in a technology possessing group that is closed (no inputs or outputs) so that the imbalance can't be transferred to another region. Or in other words, the SDGs might not be reliably achievable on a global basis without a GCI (since all communities trade). Furthermore, if GCI is required to counteract these forces of centralization for cooperation to be stable (as opposed to competition to drive unsustainable consumption), then global sustainability may not be reliably achievable without GCI either.

Though the importance of collective intelligence in achieving the SDGs has been confirmed by Ban Kimoon, former Secretary General of the UN in giving a keynote address at the World Knowledge Forum (WKF) Forum 2018 whose theme was "Collective Intelligence: Overcoming Global Pandemonium" [19], not all collective intelligence models are valid GCIs as defined within this framework. According to the reasoning presented here, collective intelligence models that are not valid GCIs as defined by this framework simply are not ensured to address this challenge of being constrained to defining the wrong problem and addressing it with the wrong solution. In creating the capacity to reliably address the optimal problem, with the optimal solution, models of GCI are then far more important as general solutions to much larger challenges than the very abstract study of GCI itself.

If, in the absence of general collective intelligence, public and private donor organizations may be driven more by incentives that centralize decision-making, then by the assumptions made in this paper, instead of driving such organizations to compete by continuously solving collective problems more and more efficiently, those incentives might in some cases effectively not be concerned with solving the problems at all. And if existing incentives are effectively unconcerned with solving the real problems, the challenge is then launching a GCI whose value proposition is significantly increasing impact, when an increase in impact doesn't in practice incentivize the organizations concerned. This reasoning creates a chicken and egg problem. Without GCI even charitable organizations might have a tendency to be constrained to compete to attain greater and greater control of philanthropic resources rather than be incentivized to create a GCI, regardless of the potential increase in outcomes. Surveys under way suggest that even conceptual case studies [16] suggesting the potential for GCI to multiply effectiveness in solving collective challenges by up to seven hundred and fifty fold do not in all cases reliably incentivize governments or donors to participate in studies of the feasibility of implementing one. For this reason, in the current centralized decision-making paradigm it may be difficult to create sufficient initial incentive for stakeholders to implement a general collective intelligence, regardless of whether doing so might be essential to actually solving the most critical social, environmental, economic, or other collective challenges globally. One potential solution to this chicken and egg problem is creating massive mind share regarding this potential for impact, so that rather than relying on alignment with the interests of any single party with sufficient resources to fund such a large scale project alone, it may be possible to fund the project through a great many smaller contributions from the much larger group with an interest in collective global well-being.

\section{Using General Collective Intelligence to Remove the Constraints to Problem Solving}

In order to remove these constraints, the group must have the collective intelligence to select the optimal problems to solve and the collective intelligence to use all available people and information as resources in solving them. This recently defined model of GCI proposes the requirements for a system to have these capabilities: 
Functional modeling (problems and solutions)

Functional decomposition

Functional domain bridging

Functional fitness

Functional stability

Functional adaptation

Functional cooperation
Components are modeled only by function to remove prejudice for or against a given implementation.

Functional components are decomposed into their most basic functional building blocks for reuse.

Different domains in which different functions are more fit in achieving the same purpose are identified. These domains are bridged by using a set of weights which identify the best function in each domain.

Every functional component is assigned some projected and actual fitness in achieving its function.

For functional components to persist they must display some degree of stability in function.

For functional components to persist in a changing environment they must have the ability to adapt their function.

Functional components must interact according to the principles of decentralized cooperation in order to maximize outcomes for each. Functions must be prioritized according to the principles of centralized cooperation in order to maximize outcomes globally.

\section{Increasing Problem Solving Ability}

Solving problems is an issue of being focused on the correct problem, having the will to solve it, and having the capability and capacity to do so. The optimal problem to solve may be constantly changing. And no single person has access to all information, or is capable of processing all information. For example, consider the case in which a donor organization issues a call for solution proposals from either of two research disciplines. Of the one hundred proposed solutions it may have received that are defined within these two fields of study, an individual program manager might be capable of reviewing the two-thirds (sixty six) that are the least complex. But the solutions formally proposed have been provided in response to only one of many potential definitions of the problem, and therefore are not all the solutions potentially available. Breaking the problem into its basic building blocks, and considering all the potential solutions in the world to each component, then rather than sixty six, there may be billions of potential solutions to evaluate. Using any single person's definition of the problem, regardless of how well-intentioned, may tend to be misaligned with the correct problem wherever the optimal problem definition is not within their knowledge or expertise. And given the total volume of the problem and solution spaces existing within the minds of the earth's seven and a half billion people, this may be most of the time.

As for having the will to solve the problem, if the true problem is the lack of a system of collective decision-making rather than any problem solvable by any single individual, the issue of "will" is extremely deceptive. It's very tempting to believe that when our choice of leader is elected to run the group, or when a leader we don't like is taken from that position, that optimal solutions will be selected. But even if a person was completely willing to address a given issue, if all efforts are constrained to solutions within their understanding, and within their capacity to evaluate, then from the above reasoning, for group problems that are large enough to be outside of the capacity of any group leader to manage, efforts must then most often be directed by that leader towards solving the wrong problem with the wrong solution. If these assumptions are true, then replacing the intelligence of one individual with that of another, rather than replacing it with a system of general collective intelligence 
with vastly greater problem solving capacity, may not necessarily dedicate the group's effort towards impact more effectively.

Furthermore, any beliefs or other cognitive biases [6] ensure that any individual, including group leaders, must systematically reject classes of solutions that don't align with their beliefs or biases. This further reduces the problem space they are willing or able to understand, and reduces the solution space they are willing or able to consider. For example, those who believe that all people are not equal in all capabilities may reject problem definitions or solutions that identify people as being equal. And those who believe that all people are equal in all capabilities may reject all people being seen as not equal. Regardless of which belief they have, if they are identified with the belief rather than identified with a system for objectively assessing the truth of what actually works to maximize outcomes, then by the reasoning given above, when it comes to understanding a large space of collective problems and solutions, they may be wrong the majority of the time.

Finally, even if a leader in a group driven by centralized decision-making has no cognitive biases against any problem definition or solution, actually orchestrating the cooperation required to maximize impact on any collective problem, or orchestrating the cooperation required to maximize impact in implementing the solution, might still be a massive logistical challenge. As an example, assume that one individuals wants to build a hospital. If that individual cooperates with one other person also wanting to build a hospital perhaps they might figure out ways to save $5 \%$. If they could cooperate with ten thousand people wanting to build a hospital perhaps they might figure out ways to save $50 \%$. If they could find ways to cooperate with everyone else in the world wanting to build a hospital, perhaps they could create enough value through this cooperation to pay the cost of building a hospital for every jurisdiction needing one. Each of the potentially billions of links in this chain of cooperation is a transaction that has to be searched for. Even if an individual leader has the will to cooperate in this way and has no cognitive biases that would block him or her from considering any given solution, finding all these potential transactions is logistically impossible without computational collective intelligence.

\section{Conclusion}

In the organization of multicellular organisms, nature has already developed massively scalable mechanisms of cooperation capable of sustainably maximizing impact for the organism as a collection of cells, on any challenges it faces. As an example, overturning a petri dish of one hundred million single-celled organisms, those organisms might not be able to travel more than a meter before needing to find new food and others resources. But by following principles of intelligent cooperation, cells might cooperate to store resources between cell boundaries in times of plenty, and cooperate to share those resources in times of need, to create the possibility for them to combine into a mouse, a bird, or other creature, that might instead have the ability to travel hundreds of kilometers to new resources, thereby increasing its sustainbility by orders of magnitude. This paper has proposed that simple functional models might be used to mimic that intelligent cooperation so it can be used to increase the sustainability of human processes by orders of magnitude in the same way. And this paper has proposed that these simple functional models might be used to make cooperation radically more robust and stable againist any possible non-cooperation or other disruption. Since if this model is valid in representing the mechanisms of cooperation that exist in nature, then these mechanisms are ones nature has spent 3.5 billion years perfecting. The robustness and sustainability of these mechanisms might be far greater than any ones human beings might create.

In nature, these mechanisms of cooperation are proposed to have the capacity to sustainably maximize impact on any challenges faced by organisms as a collection of cells. When replicated in human 
groups, they are proposed to have the capacity to sustainably maximize the group's impact on any collective challenges as well, including sustainability and sustainable development. A General Collective Intelligence (GCI) as defined within this framework simply implements a model of these mechanisms with the aim of organizing group decision-making so this sustainable maximization of impact on collective challenges for groups is reliably achievable.

This model of GCI is proposed to be a mathematical solution to the problem of achieving stability in the collective ability to solve problems, despite potentially chaotic changes in the problems themselves [8]. Since this stability of function in problem solving is defined by stability in fitness to execute the functions of the collective, which is in turn defined by the collective well-being [7], a GCI may be a mathematical solution to sustainably maximizing impact on all challenges to collective well-being, including the SDGs.

\section{References}

[1] The Relationship Between Collective Intelligence and One Model of General Collective Intelligence, Andy E. Williams, Computational Collective Intelligence, 11th International Conference, ICCCI 2019, Hendaye, France, September 4-6, 2019, Proceedings, Part II, Pages 589-600

[2] Cartledge, K., C. Dürrwächter, V. Hernandez Jimenez, and N. P. Winder. 2009. Making sure you solve the right problem. Ecology and Society 14(2): r3. [online] URL:

http://www.ecologyandsociety.org/vol14/iss2/resp3/

[3] Cockerill K., Armstrong M., Richter J., Okie J.G. (2017) Integrating Science and Society for Environmental Realism. In: Environmental Realism. Palgrave Macmillan, Cham

[4] Newell B, Proust K (2012) Escaping the complexity dilemma. Australian National University digital repository, Canberra

[5] Andy E. Williams, The Principles Enabling Cooperation within Conscious Organisms and Collectives, working paper.

[6] Arnott, D. (2006), Cognitive biases and decision support systems development: a design science approach. Information Systems Journal, 16: 55-78. doi:10.1111/j.1365-2575.2006.00208.x

[7] Andy E. Williams, The Functional Modeling Framework as a Tool for Defining and Comparing Models of Consciousness or Cognition, under review

[8] Andy E. Williams, A Model for General Collective Intelligence, under review

[9] Goodin, R., Moran, M., Rein, M., \& Friedman, B. (2008-06-12). Policy Analysis as Organizational Analysis. In The Oxford Handbook of Public Policy. : Oxford University Press. Retrieved 25 Jan. 2020, from https://www.oxfordhandbooks.com/view/10.1093/oxfordhb/9780199548453.001.0001/oxfordhb9780199548453-e-023.

[10] Goodin, R., Moran, M., Rein, M., \& Immergut, E. (2008-06-12). Institutional Constraints on Policy. In The Oxford Handbook of Public Policy. : Oxford University Press. Retrieved 25 Jan. 2020, from https://www.oxfordhandbooks.com/view/10.1093/oxfordhb/9780199548453.001.0001/oxfordhb9780199548453-e-027.

[11] Robert Lempert, Agent-based modeling as organizational and public policy simulators Proceedings of the National Academy of Sciences May 2002, 99 (suppl 3) 7195-7196;

DOI:10.1073/pnas.072079399

[12] Morgan, M. Granger, Max Henrion and Mitchell Small. "Uncertainty: A Guide to Dealing with Uncertainty in Quantitative Risk and Policy Analysis.” (1990).

[13] Conte, R., Gilbert, N., Bonelli, G. et al. Eur. Phys. J. Spec. Top. (2012) 214: 325.

https://doi.org/10.1140/epjst/e2012-01697-8

[14] Stone, Deborah A. "Causal Stories and the Formation of Policy Agendas." Political Science Quarterly, vol. 104, no. 2, 1989, pp. 281-300. JSTOR, www.jstor.org/stable/2151585. Accessed 25 Jan. 2020 . 
[15] Goda, Thomas. (2016). Global trends in relative and absolute income inequality. Ecos de Economía, 20(42), 46-69. https://dx.doi.org/10.17230/ecos.2015.42.3

[ conceptual case studies showing the potential to multiply effectiveness in solving collective challenges by up to seven hundred and fifty fold]

[16] Andy E. Williams, The Collective Intelligence based Program to Accelerate Achievement of the Sustainable Development Goals as a Case Study for Collectively Intelligent Program Design, working paper

[17] Schönfeldt, H., \& Gibson Hall, N. (2012). Dietary protein quality and malnutrition in Africa. British Journal of Nutrition, 108(S2), S69-S76. doi:10.1017/S0007114512002553

[18] Hall, Roger I., The Natural Logic of Management Policy Making: Its Implications for the Survival of an Organization, Management Science (1984), volume 30, number 8, pages 905-927, https://doi.org/ 10.1287/mnsc.30.8.905

[19] "Co-chair Ban Ki-moon delivers keynote address at the World Knowledge Forum 2018", https://bankimooncentre.org/tag/collective-intelligence , Published October 15, 2018, Retrieved Jan 26, 2020.

Declarations

Ethics approval and consent to participate:

Not applicable.

Consent for publication

Not applicable.

\section{Availability of data and material}

All data generated or analysed during this study are included in this published article.

\section{Competing interests}

The authors declare that they have no competing interests.

\section{Funding}

Not applicable.

\section{Authors' contributions}

The article was authored entirely by Andy E. Williams

\section{Acknowledgements}

Not applicable. 